\title{
UTILIZAÇÃO DE MÉTODOS DE REPRESENTAÇÃO ESPACIAL PARA CÁLCULO DO FATOR TOPOGRÁFICO NA EQUAÇÃO UNIVERSAL DE PERDA DE SOLO REVISADA EM BACIAS HIDROGRÁFICAS ${ }^{(1)}$
}

\author{
Jean Paolo Gomes Minella ${ }^{(2)}$, Gustavo Henrique Merten $^{(3)} \&$ \\ Anderson Luis Ruhoff ${ }^{(4)}$
}

\begin{abstract}
RESUMO
Métodos de representação espacial para o cálculo do fator topográfico (LS) da Equação Universal de Perda de Solo Revisada (RUSLE) têm sido utilizados para estimar a erosão do solo e a produção de sedimentos em bacias hidrográficas. Esses procedimentos baseiam-se nas equações tradicionalmente empregadas para determinação do fator LS e em informações que caracterizam a forma das vertentes derivadas do modelo numérico de elevação (MNE). Neste estudo foram analisados dois métodos de representação espacial utilizados no cálculo do fator LS em modelos matemáticos de erosão e produção de sedimentos em bacias hidrográficas. A análise foi realizada em quatro bacias rurais de relevo movimentado. Os valores de LS obtidos pelos métodos de representação espacial foram comparados entre si e com valores de LS determinados pelo método tradicional com levantamento em campo. Resultados mostraram que os valores de LS gerados pelos métodos de representação espacial apresentam diferenças significativas entre si, sendo dependentes do procedimento de cálculo e do método utilizado para determinar a direção de fluxo no MNE. Também foi verificado que os valores numéricos do fator LS determinados pelos métodos de representação espacial apresentam diferenças em relação àqueles estimados pelo método tradicional.
\end{abstract}

Termos de indexação: Equação Universal de Perda de Solo Revisada, produção de sedimentos, erosão de encostas, modelagem numérica do terreno, sistema de informação geográfica.

\footnotetext{
(1) Recebido para publicação em fevereiro de 2009 e aprovado em junho de 2010.

(2) Professor Adjunto do Departamento de Solos, Universidade Federal de Santa Maria - UFSM. Av. Roraima 1000, Camobi, CEP 97105-900 Santa Maria (RS). E-mail: jminella@smail.ufsm.br

(3) Professor do Instituto de Pesquisas Hidráulicas, Universidade Federal do Rio Grande do Sul - UFRGS. Av. Bento Gonçalves 7712, CEP 91540-000 Porto Alegre (RS). Bolsista do CNPq. E-mail: merten@iph.ufrgs.br

(4) Doutorando do Programa de Pós-Graduação em Recursos Hídricos e Saneamento Ambiental, Instituto de Pesquisas Hidráulicas, Universidade Federal do Rio Grande do Sul - UFRGS. Av. Bento Gonçalves 7712, CEP 91540-000 Porto Alegre (RS). Bolsista do CNPq. E-mail: anderson.ruhoff@ufrgs.br
} 


\title{
SUMMARY: USE OF SPATIAL REPRESENTATION TO CALCULATE THE TOPOGRAPHIC FACTOR IN THE REVISED UNIVERSAL SOIL LOSS EQUATION IN WATERSHEDS
}

\begin{abstract}
Methods of spatial representation to calculate the topographic factor $(L S)$ of the Revised Universal Soil Loss Equation (USLE) have been used to estimate soil erosion and sediment yield of watersheds. These procedures are based on equations traditionally used to determine the LS factor and information that characterize the hillslope forms and processes, derived from the Digital Elevation Model (DEM). Two computational methods commonly used to calculate LS factor in soil erosion and sediment yield models were analyzed in this study. The analysis was performed in four small rural watersheds with hilly terrain. The LS values obtained by the methods of spatial representation were compared with each other with LS values measured by the traditional method. Results show significant differences of the computergenerated LS values, which were related to the calculation procedure and the method used to determine the DEM flow direction. Differences were also observed between the numeric values of the LS factor determined by methods of spatial representation and in the field.
\end{abstract}

Index terms: Revised Universal Soil Loss Equation, sediment yield, hillslope erosion, numeric terrain modeling, geographic information systems.

\section{INTRODUÇÃO}

A Equação Universal de Perda de Solo - USLE (Wischmeier \& Smith, 1978) e mais tarde a sua versão revisada - RUSLE (Renard et al., 1997) foram desenvolvidas com o propósito de estimar a erosão hídrica a partir de variáveis climáticas (fator $\mathrm{R}$ ), pedológicas (fator K), topográficas (fator LS) e das condições de uso, manejo e conservação de solo (fator CP). Ainda que a escala de aplicação da USLE/ RUSLE seja para encostas curtas, existem variantes destas que têm sido utilizadas para estimar a erosão e a produção de sedimentos na escala de bacias hidrográficas (Merrit et al., 2003). Esse é o caso da Equação Modificada de Perdas de Solo - MUSLE (Willians, 1975) e de modelos que calculam a produção de sedimentos utilizando a combinação da USLE com a razão de distribuição de sedimentos (Sediment Delivery Ratio). A razão de distribuição de sedimentos representa uma fração de todo o material que foi erodido pelos processos erosivos e transferido para o exutório da bacia ou uma dada seção transversal do canal aluvial (Walling, 1983; Hadley et al., 1985). Entre os modelos que utilizam esse procedimento, destacam-se o Agricultural Non-Point Source Model AGNPS (Young et al., 1989), Areal Non-Point Source Watershed Environment Response Simulation ANWERS (Moore \& Gallant, 1991), USLE-M (Kinnell \& Risse, 1998) e Soil Water Assessment Tool - SWAT (Arnold et al., 1990).

O fator LS combina os fatores comprimento da rampa L com declividade S. A determinação desse fator apresenta limitações em áreas de relevo complexo ou de grandes extensões, levando com isso a estimativas erradas das taxas de erosão do solo.
Situações comuns de aplicações equivocadas não levam em consideração que o fator LS representa a contribuição do escoamento superficial no processo de erosão hídrica. Nesse contexto, o fator L representa uma dimensão linear que corresponde teoricamente a uma distância que vai do início da formação do escoamento (condição de escoamento Hortoniano) até um ponto onde esse encontra um canal ou uma condição que favoreça a deposição dos sedimentos (Renard et al., 1997). Na prática, essa determinação não é simples, devido à dificuldade de identificar o início da formação do escoamento e dos locais de deposição. Essa situação torna impraticável o cálculo tradicional do fator LS em áreas de grande extensão e de relevo complexo. Nesse sentido, uma importante contribuição para a automatização do modelo USLE/ RUSLE foi a incorporação, no fator topográfico LS, dos conceitos de potência do escoamento, área de contribuição específica e técnicas de geoprocessamento.

Atualmente, esses conceitos têm sido incorporados em métodos de representação espacial, permitindo calcular o fator LS para grandes áreas e de relevo complexo, como em bacias hidrográficas. Este trabalho teve o propósito de avaliar a utilização de dois métodos de representação espacial para o cálculo do fator LS; a análise compara os dois métodos entre si e estes com os valores medidos no campo e calculados de forma tradicional.

\section{MATERIAL E MÉTODOS}

\section{Cálculo do fator topográfico (LS)}

$\mathrm{O}$ fator $\mathrm{L}$, quando determinado para vertentes de relevo uniforme (encosta retilínea) na versão RUSLE 
(Renard et al., 1997), é definido matematicamente pela equação 1 (McCool et al., 1989).

$$
L=\left(\frac{\lambda}{\lambda_{u}}\right)^{m}
$$

em que $\lambda$ é a distância equivalente à projeção horizontal de uma encosta; $\lambda_{\mathrm{u}}$ corresponde ao comprimento longitudinal da parcela-padrão $(22,13 \mathrm{~m})$ utilizada para obtenção dos parâmetros da USLE; e "m" é um expoente que incorpora a relação entre a participação do processo de erosão em sulco e entressulco (Foster et al., 1977; McCool et al., 1989, 1997).

Já o fator S da RUSLE é definido de acordo com McCool et al. (1997) pelas equações 2 e 3.

$$
\begin{aligned}
& S=10,8 \operatorname{sen} \theta+0,03 \text { para } \tan \theta<0,09 \\
& S=16,8 \operatorname{sen} \theta-0,50 \text { para } \tan \theta \geq 0,09
\end{aligned}
$$

em que $\theta$ representa o ângulo da encosta.

$\mathrm{Na}$ determinação do fator L para condições de encostas com relevo não retilíneo, o comprimento da vertente é segmentado em trechos com declividades uniformes, sendo estimado de acordo com a equação 4 (Foster \& Wischmeier, 1974).

$$
L=\left[\frac{\lambda_{i}^{m+1}-\lambda_{i-1}^{m+1}}{\left(\lambda_{i}-\lambda_{i-1}\right) \times 22,13^{m}}\right]
$$

em que $\lambda_{\mathrm{i}}$ e $\lambda_{\mathrm{i}-1}$ (em metros) representam os trechos inicial e final do segmento de declive uniforme.

No caso da utilização da MUSLE, o fator LS passa a se constituir em um fator bidimensional, pois o $\mathrm{L}$ deixa de ter uma dimensão linear para assumir uma dimensão de área ou uma unidade hidrológica representativa da bacia. O conceito de unidade hidrológica incorpora a noção de uma área uniforme onde ocorre a formação do escoamento superficial. Nessas condições, a determinação do fator L torna-se mais difícil devido à complexidade do relevo de uma bacia hidrográfica.

Antes do advento das técnicas de geoprocessamento, foram propostos diferentes métodos para estimar o fator LS para bacias. Williams \& Berndt (1977), por exemplo, avaliaram diferentes procedimentos utilizando relações que levavam em consideração a área de drenagem, a densidade de drenagem e o comprimento das linhas de contorno e dos canais de drenagem de uma bacia.

Com as técnicas disponíveis dos Sistemas de Informações Geográficas (SIG) e a facilidade de obtenção de Modelos Numéricos de Elevação (MNE), tem sido possível estimar o fator LS de forma menos laboriosa, levando-se em conta as feições geomorfológicas do relevo, que são determinantes em relação aos processos hidrológicos que ocorrem em áreas de relevo complexo.

Moore \& Burch (1986a,b,c) propuseram uma base teórica para determinação do fator LS pela incorporação da teoria da potência unitária do escoamento, apresentada por Yang $(1972,1973,1984)$. De acordo com essa teoria, a água na superfície do solo apresenta determinada energia capaz de desagregar e transportar partículas de solo quando estas se movem no sentido do declive. Esse conceito, combinado com as equações 1,2 e 3 do cálculo tradicional do fator LS, derivou métodos que são utilizados para representar espacialmente o fator LS em áreas de relevo complexo, como em bacias hidrográficas - por exemplo, aqueles baseados em Moore \& Burch (1986a) e em Desmet \& Govers (1996). A equação 5 representa essa nova conceituação utilizada para estimar o fator LS.

$$
L S_{p}=\left(\frac{a \cdot l}{22,13}\right)^{0,4}\left(\frac{s}{0,0896}\right)^{1,3}
$$

em que $\mathrm{LS}_{\mathrm{p}}$ representa o fator LS derivado da teoria da potência do escoamento; $a$, o fator de forma da vertente (Equação 6); $l$, a distância longitudinal da unidade de contribuição hidrológica; e $s$, o gradiente de declividade.

O valor do denominador do segundo membro da equação 5 refere-se ao seno do ângulo de $9 \mathrm{~cm} \mathrm{~m}^{-1}$, que corresponde à declividade da parcela-padrão de onde inicialmente foi derivado o fator LS. O fator de forma $a$ é de grande importância para representação dos processos hidrológicos de superfície. Quando $a=1$, a forma da unidade hidrológica é retilínea; quando $a>1$, a forma da unidade é convergente; e quando $a<1$, a forma representa condição de divergência. Áreas de convergência, por exemplo, podem ser consideradas preferenciais para formação do escoamento em uma bacia; teoricamente, nessas áreas o escoamento superficial apresentaria uma condição de maior potência hidráulica para gerar trabalhos relativos à desagregação e ao transporte de sedimentos, quando comparado com unidades hidrológicas de divergência $(a<1)$. A curvatura das vertentes, no plano e no perfil, condiciona sensivelmente o comportamento dos processos erosivos. O fator de forma $a$ é calculado pela razão entre a área da unidade de contribuição hidrológica $(A)$ e o produto da largura da seção transversal da unidade hidrológica no seu exutório (b) pela distância longitudinal da unidade de contribuição hidrológica (l) (Equação 6).

$$
a=\frac{A}{b \cdot l}
$$

O produto de " $a$ " e " $l$ ", numerador do primeiro termo da equação 5 , também é conhecido como área 
de contribuição específica $\left(\mathrm{A}_{\mathrm{s}}\right)$, definida também como a área acumulada de drenagem dividida pela largura da seção transversal da unidade hidrológica no seu exutório $\left(A_{s}=A / b=a l\right)$ (Onstad \& Brakensiek, 1968). Para vertentes convexas, em que o fluxo acumulado torna-se divergente, a área de contribuição específica tende a diminuir. Para vertentes côncavas, a área de contribuição específica tende a aumentar, causando rápido incremento no fluxo acumulado.

O valor da área de contribuição hidrológica $(A)$ é obtido com o uso de um SIG a partir do modelo numérico de elevação; para isso, o SIG necessita realizar uma etapa intermediária, que é o cálculo da direção de fluxo em cada célula (Moore et al., 1991). A descrição da direção de fluxo entre as células do MNE pode ser de dois tipos: fluxo único e fluxo múltiplo. No primeiro caso, apenas uma direção é estabelecida para cada célula, e esse valor é na direção da célula vizinha de cota mais baixa. Entretanto, é importante considerar que os fluxos entre as células quase nunca correspondem a uma única direção, pois uma célula pode receber o fluxo proveniente de várias células e transferir o fluxo acumulado para várias outras células. Dessa forma, Quinn (1991) propôs a direção de fluxo múltiplo (MS-Quinn - multiple directions based on slope), em que o percentual de fluxo transferido para cada célula a jusante é proporcional ao produto da distância de fluxo acumulado e ao fator de peso geométrico, o qual depende da direção de fluxo. Essa foi uma importante contribuição para a representação do escoamento superficial em estudos que necessitam representar o fluxo difuso, como os de estudos de escoamento superficial e erosão.

Os conceitos que relacionam fluxo acumulado e a forma das vertentes aos processos erosivos (Equações 5 e 6) contribuem para a modelagem matemática da erosão em bacias, uma vez que o método tradicional (Equações 1 a 4) não considera a complexidade do relevo. Esses conceitos têm sido incorporados em modelos matemáticos no cálculo do fator LS para bacias hidrográficas.

\section{Localização e caracterização das áreas de estudo}

Para execução deste trabalho, foram utilizadas quatro sub-bacias, que estão inseridas na bacia do Arroio Lajeado Ferreira (28 ${ }^{\circ} 49^{\prime} 30$ " S e $52^{\circ} 12^{\prime} 15$ ” O) (Figura 1). A bacia do Arroio Lajeado Ferreira possui uma área aproximada de 119 ha, característica da região de cabeceira do rio TaquariAntas, localizada na encosta superior do nordeste do Estado do Rio Grande do Sul, município de Arvorezinha. A formação geológica da bacia é caracterizada por derrames basálticos, com presença de diques e sills de diabásio, compondo o Grupo São Bento da Formação Serra Geral. O relevo apresenta elevadas declividades, em vertentes curtas e vales encaixados. Os solos predominantes são Argissolo, Cambissolo e Neossolo, cujo principal uso do solo é a cultura do fumo (Minella et al., 2007).
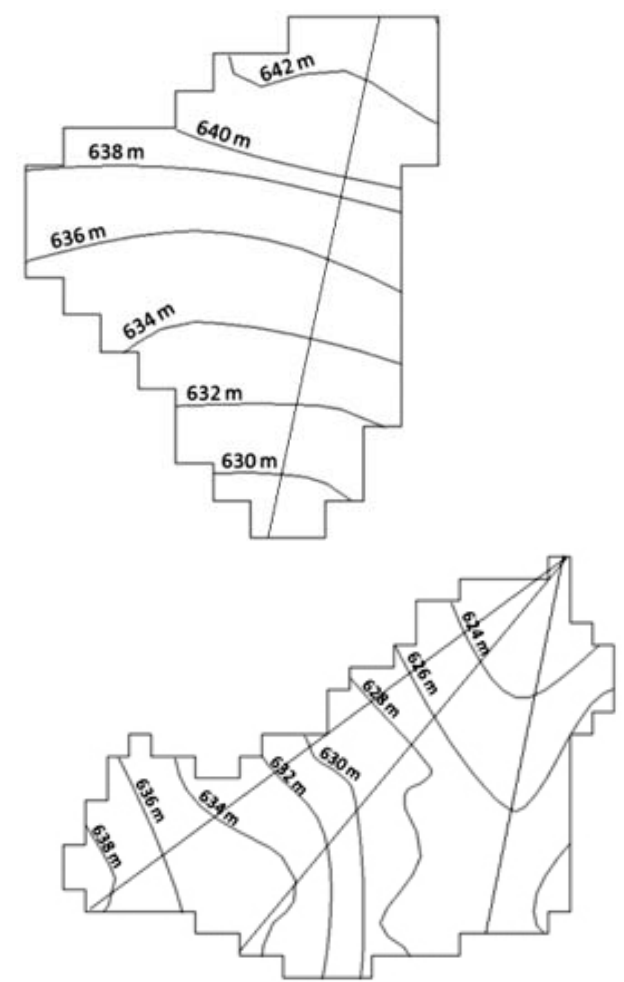

Figura 1. Curvas de nível das quatro sub-bacias analisadas (1 a 4, da esquerda para direita) - as linhas transversais às curvas de nível são os segmentos utilizados para determinação do fator LS pelo método tradicional com levantamento em campo.

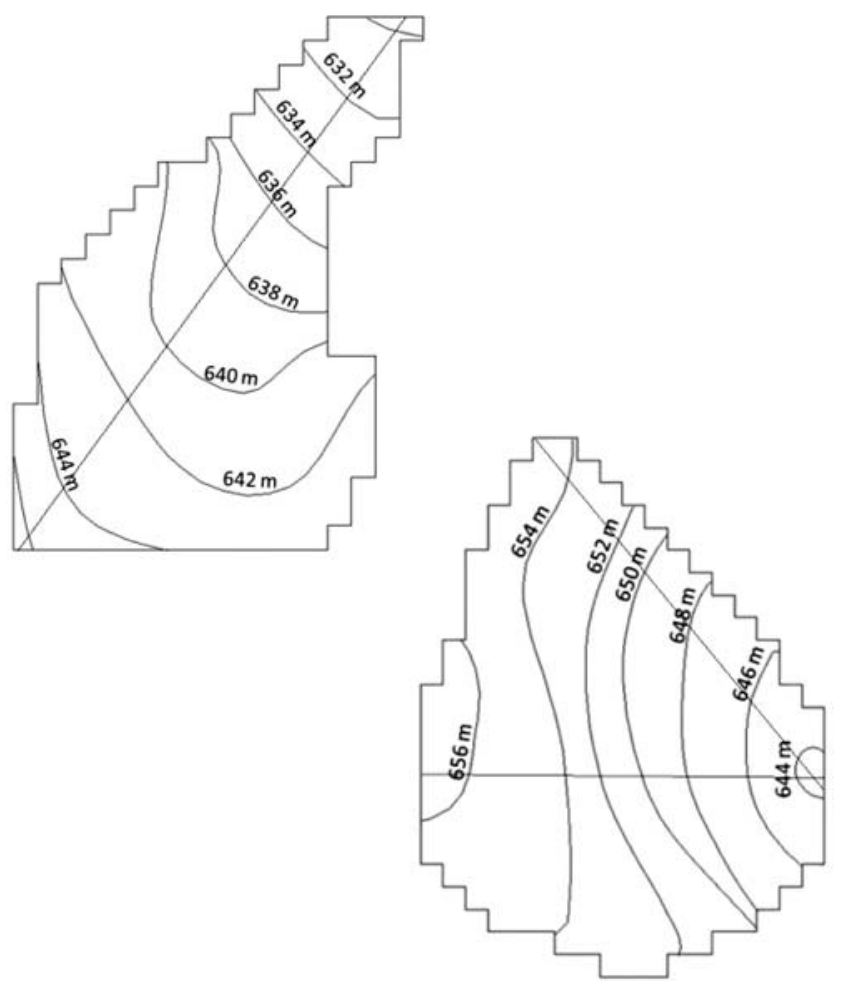




\section{Métodos utilizados para o cálculo do fator LS}

Na determinação do fator LS foram utilizados MNEs obtidos a partir de levantamentos topográficos realizados em campo. Os MNEs com $5 \mathrm{~m}$ de resolução espacial foram processados no programa SPRING. Foram utilizados 463 pontos de controle altimétrico, obtidos a partir da utilização de uma estação total, dos quais 72 localizam-se na sub-bacia $1 ; 127$, na subbacia 2 ; 160, na sub-bacia 3 ; e 104, na sub-bacia 4 . As principais características topográficas das microbacias do Arroio Lajeado Ferreira, medidas a partir do modelo numérico do terreno, estão descritas no quadro 1.

Quadro 1. Características topográficas das subbacias do Arroio Lajeado Ferreira, RS

\begin{tabular}{lrrrc}
\hline \multicolumn{1}{c}{ Sub-bacia } & $\mathbf{0 1}$ & $\mathbf{0 2}$ & $\mathbf{0 3}$ & $\mathbf{0 4}$ \\
\hline & 2.450 & 5.700 & 6.600 & 7.400 \\
$\begin{array}{l}\text { Área (m²) } \\
\text { Comprimento máximo }\end{array}$ & 65,5 & 135,4 & 134,2 & 89,0 \\
$\begin{array}{l}\text { de encosta (m) } \\
\text { Largura máxima }\end{array}$ & 50,1 & 74,7 & 65,9 & 121,0 \\
da bacia (m) & 636,5 & 639,9 & 629,3 & 651,6 \\
Altitude média (m) & 629,5 & 630,0 & 622,0 & 643,7 \\
Altitude mínima (m) & 643,0 & 647,0 & 638,5 & 657,0 \\
Altitude máxima (m) & 17,94 & 13,44 & 13,32 & 14,15 \\
Declividade média (\%) & 17,0 & & \\
\hline
\end{tabular}

Foram utilizados dois métodos de representação espacial para o cálculo do fator LS nas sub-bacias: Desmet \& Govers (1996) e Moore \& Burch (1986a). O primeiro requer a definição da área de fluxo acumulado (A), enquanto o de Moore \& Burch (1986a) requer a definição da área de contribuição específica $\left(A_{s}\right)$. A partir do Modelo Numérico de Elevação, determinouse (1) a direção de fluxo, (2) a área de fluxo acumulado, (3) a área de contribuição específica e (4) o mapa de declividade, utilizando as opções de cálculo disponíveis no programa SPRING. O método de determinação dessas informações para a modelagem ambiental está descrito em Moore et al. (1991).

Para obtenção do fator LS proposto por Moore \& Burch (1986a), aplicaram-se as equações 5 e 6, descritas anteriormente. Em se tratando do fator LS proposto por Desmet \& Govers (1996), foi utilizada a equação 7, para obtenção do fator comprimento de encostas L, e a equação 8, para obtenção do fator S. $\mathrm{O}$ fator LS corresponde ao produto dos dois fatores, resultado da equação 7 multiplicado pelo resultado da equação 8.

$$
\begin{gathered}
L_{i j}=\frac{\left[\left(A_{i j}+D_{i j}^{2}\right)^{m+1}-A_{i j}^{m+1}\right]}{\left.\left[D^{m+2}\left(\operatorname{sen} \theta_{i j}+\cos \theta_{i j}\right)^{m}(22,13)^{m}\right)\right]} \\
S_{i j}=65,41 \cdot \operatorname{sen} \theta_{i j}+4,56 \cdot \operatorname{sen} \theta_{i j}+0,065
\end{gathered}
$$

em que os subíndices $i$ e $j$ são as coordenadas da célula que a localiza no mapa da bacia; $A_{i, j}$ corresponde à área de fluxo acumulado (área de contribuição) para cada célula com coordenadas $(\mathrm{i}, \mathrm{j}) ; D$ corresponde à resolução da grade de células; $\alpha_{i, j}$ corresponde ao ângulo da direção do fluxo que indica a direção para onde o fluxo se direciona em relação ao norte; $m$ corresponde ao expoente do comprimento de encostas; e $\theta_{i, j}$ corresponde à declividade média da vertente.

Diferentemente do método tradicional, as equações 5 a 8 são aplicadas para cada célula do modelo numérico de elevação. A partir dos valores de elevação no MNE é determinado o valor de declividade, de direção de fluxo, da área acumulada e da área de contribuição específica para cada célula. Dessa forma, os resultados do fator LS obtidos para cada sub-bacia, pelos métodos de representação espacial, fornecem um conjunto de valores de LS distribuídos na área conforme a variabilidade da elevação do terreno. A análise dos resultados (conjunto de valores) foi feita por meio de estatísticas univariadas, como média, mediana, valor mínimo e valor máximo.

Um terceiro método utilizado foi a determinação do fator LS a partir de levantamento em campo dos comprimentos de rampa e declividade em transectos de cada sub-bacia. A medição foi realizada com uso de trena e clinômetro; para cada sub-bacia foram selecionadas uma ou mais rampas representativas, seguindo-se o critério teórico da representação de um segmento. Um segmento compreende desde o início da formação do escoamento até o final da encosta, determinado pela presença de um canal ou uma superfície de deposição. Nas rampas não retilíneas, estas foram divididas em segmentos de declividade uniforme. A partir dessas informações de campo, foram aplicadas as equações de 1 a 4 para calcular o fator LS em campo para cada sub-bacia.

\section{Análise estatística}

A análise estatística determinou as diferenças entre os dois métodos - Moore \& Burch (1986a) e Desmet \& Govers (1996) - de representação espacial do fator LS. Foi calculada a diferença encontrada para cada célula entre os dois métodos, utilizando o teste " $t$ " de comparação entre médias para amostras pareadas (dependentes) (Davis, 1986). Este teste foi escolhido porque é possível comparar as células (pixels) em cada posição e não apenas a média geral das células na bacia.

Como os resultados, de ambos os métodos, é uma matriz de mesmo tamanho e posição geográfica, o valor de LS obtido em cada célula por um dos métodos é comparado com seu respectivo par no outro método, determinando a diferença entre os valores de cada ponto. A partir dessa matriz, que mostra o valor das diferenças entre os métodos, é calculada a média $\left(\mu_{0}\right)$, e para esta é testada a hipótese nula de que esse valor é igual a zero $\left(\mathrm{H}_{0}: \mu_{0}=0\right)$, ou seja, que não existe diferença significativa entre os métodos, ou, no caso 
de rejeição da hipótese nula, que o valor das diferenças é significativo $\left(\mathrm{H}_{1}: \mu_{0} \neq 0\right)$. Esse método permitiu avaliar as diferenças entre os dois métodos, considerando a variabilidade encontrada dentro das sub-bacias para o valor do fator LS. A comparação dos métodos de representação espacial com o método tradicional de determinação em campo foi feita utilizando a média dos valores de LS das células da bacia para os métodos de representação espacial com o valor encontrado no segmento para o método tradicional.

\section{RESULTADOS E DISCUSSÃO}

\section{Comparação entre os métodos de representação espacial}

A distribuição espacial do fator LS calculado pelos métodos de Desmet \& Govers (1996) e Moore \& Burch (1986a), utilizando o algoritmo de fluxo múltiplo presente no SPRING, é apresentada nas figuras 3 e 4. Observa-se que a distribuição espacial das classes do fator LS apresenta padrão semelhante entre os dois procedimentos. Tanto o método de Desmet \& Govers (1996) como o de Moore \& Burch (1986a) foram capazes de representar espacialmente as diferenças no potencial erosivo do escoamento, como pode ser observado nas figuras 2 e 3, em comparação com a figura 1, onde são apresentadas as curvas de nível das quatro sub-bacias analisadas.

No quadro 2 é apresentado o resumo do fator LS obtido a partir da aplicação dos métodos propostos por Desmet \& Govers (1996) e Moore \& Burch (1986a). A primeira evidência é de que os valores do fator LS são maiores quando utilizado o método de Desmet \& Govers (1996) em aproximadamente uma unidade para os valores médios. Os resultados da análise estatística mostraram que, para todas as sub-bacias, a diferença média entre células foi significativa a 99,99\%.

Essas diferenças ocorrem principalmente em função do uso de conceitos distintos de cálculo da área acumulada de montante. Enquanto o método de Desmet \& Govers (1996) utiliza o conceito de fluxo acumulado de drenagem a montante, o de Moore \& Burch (1986a) emprega o conceito de área de contribuição específica, que resulta em valores mais baixos de fluxo acumulado de células de montante. Assim, quando os valores de fluxo de montante são elevados, o método de Desmet \& Govers (1996) tende a estimar também valores elevados do fator LS, especialmente nas posições próximas à drenagem, ao passo que essa condição não é verificada quando usado o método de Moore \& Burch (1986a). Verifica-se também que os valores de LS representados pela média (Quadro 2) são superiores quando comparados com os

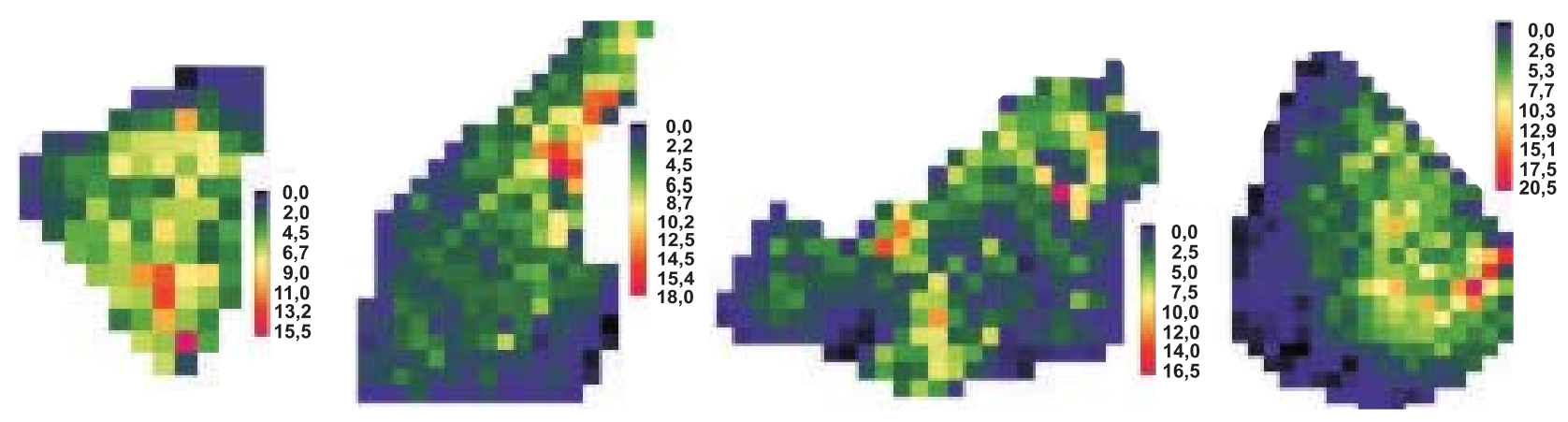

Figura 2. Fator LS calculado pelo método de Desmet \& Govers (1996) para as quatro bacias.
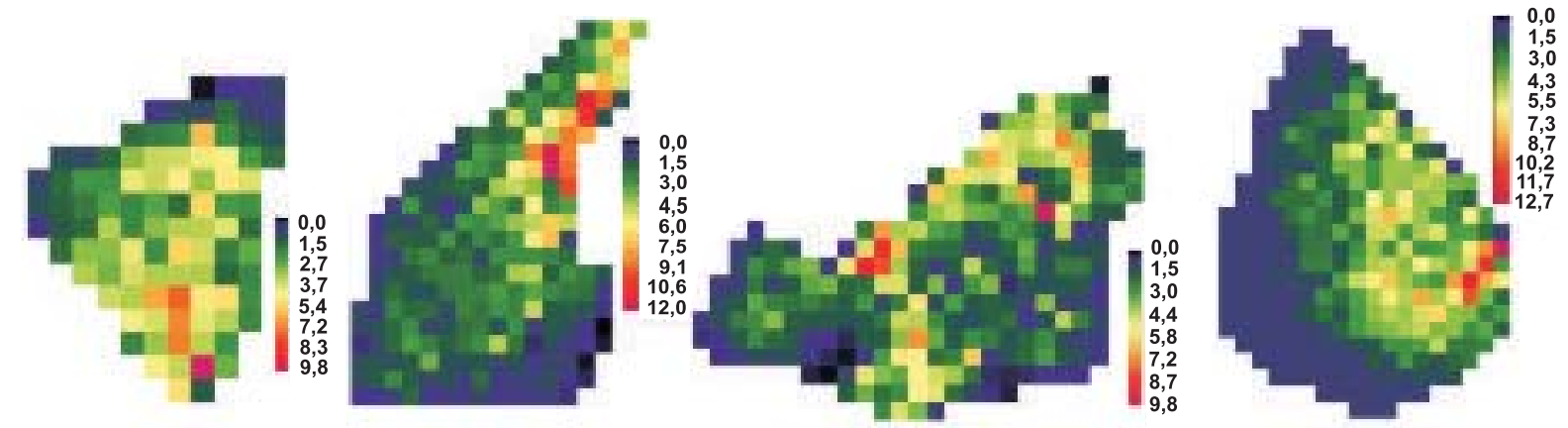

Figura 3. Fator LS calculado pelo método de Moore \& Burch (1986a) para as quatro bacias. 
de LS representados pela mediana, uma vez que a média é influenciada pelos valores máximos, enquanto a mediana não.

Comparação dos métodos de representação espacial com o método tradicional de levantamento em campo

Os resultados dos valores de LS determinados por meio das medições de campo são apresentados no quadro 3. Verifica-se que o fator LS variou de um mínimo de 2,33, para uma das rampas medidas na sub-bacia 4, até um máximo de 6,02, para uma das rampas medidas na sub-bacia 3 . Em termos de grandeza numérica, os valores de LS determinados em campo podem ser considerados de médio a alto e representativos de relevo declivoso.

Comparando os valores de LS do quadro 3 (método tradicional) com os obtidos pelos métodos de representação espacial (Quadro 2), verifica-se que os valores de LS encontram-se na mesma ordem de grandeza; para todas as sub-bacias, os valores do método tradicional com levantamento em campo são estatisticamente diferentes do valor médio encontrado nos métodos de representação espacial. Nas sub-bacias onde se mediu mais de um segmento foi utilizado o valor médio para a comparação. Salienta-se que para a sub-bacia 3 os valores de LS (6,02, 4,75 e 4,49) são ligeiramente superiores aos das médias e medianas dos valores de LS pelos métodos de representação espacial. É importante salientar que há ganho significativo de informação nos métodos de representação espacial, já que o resultado é obtido para cada unidade de área (célula), tendo-se assim a variabilidade bidirecional do fator, ao passo que no método tradicional com levantamento em campo os valores correspondem a uma ou mais medidas unidirecionais, consideradas como representativas da realidade da área.

\section{CONCLUSÕES}

1. Os resultados indicam que o uso de métodos de representação espacial de cálculo do fator LS permite estimar a distribuição espacial do fator topográfico em áreas de relevo complexo, contribuindo significativamente para a aplicação de modelos do tipo USLE/ RUSLE na escala de bacia hidrográfica.

2. O método proposto por Desmet \& Govers (1996) gerou resultados maiores do que aqueles gerados por Moore \& Burch (1986a). A razão para isso é que o primeiro usa o conceito de fluxo acumulado de drenagem a montante, e o segundo, o conceito de área

Quadro 2. Estatísticas do fator LS para as quatro sub-bacias, calculadas com base nos métodos de (1) Desmet \& Govers (1996) e (2) Moore \& Burch (1986a)

\begin{tabular}{|c|c|c|c|c|c|c|c|c|}
\hline & \multicolumn{2}{|c|}{ Sub-Bacia 1} & \multicolumn{2}{|c|}{ Sub-Bacia 2} & \multicolumn{2}{|c|}{ Sub-Bacia 3} & \multicolumn{2}{|c|}{ Sub-Bacia 4} \\
\hline & 1 & 2 & 1 & 2 & 1 & 2 & 1 & 2 \\
\hline Máximo & 16,17 & 10,20 & 18,42 & 12,16 & 17,89 & 9,94 & 20,72 & 12,73 \\
\hline Mínimo & 0,28 & 0,13 & 0,20 & 0,07 & 0,23 & 0,09 & 0,16 & 0,04 \\
\hline Mediana & 4,13 & 2,92 & 2,72 & 2,16 & 2,45 & 1,78 & 2,98 & 2,23 \\
\hline Média & 4,76 & 3,24 & 3,59 & 2,74 & 3,29 & 2,34 & 3,87 & 2,71 \\
\hline Desvio-padrão & 2,94 & 1,85 & 2,99 & 2,24 & 2,67 & 1,74 & 3,34 & 2,20 \\
\hline Núm. células & \multicolumn{2}{|c|}{98} & \multicolumn{2}{|c|}{229} & \multicolumn{2}{|c|}{265} & \multicolumn{2}{|c|}{297} \\
\hline Dif. med. & \multicolumn{2}{|c|}{1,52} & \multicolumn{2}{|c|}{0,85} & \multicolumn{2}{|c|}{0,95} & \multicolumn{2}{|c|}{1,16} \\
\hline Teste $t$ & \multicolumn{2}{|c|}{13,10} & \multicolumn{2}{|c|}{14,88} & \multicolumn{2}{|c|}{14,24} & \multicolumn{2}{|c|}{15,92} \\
\hline Prob. $\mathrm{H}_{0}$ & \multicolumn{2}{|c|}{$<0,0001$} & \multicolumn{2}{|c|}{$<0,0001$} & \multicolumn{2}{|c|}{$<0,0001$} & \multicolumn{2}{|c|}{$<0,0001$} \\
\hline
\end{tabular}

Quadro 3. Resultados da determinação do fator LS medido em campo nas quatro sub-bacias do Arroio Lajeado Ferreira, RS

\begin{tabular}{|c|c|c|c|c|}
\hline Sub-bacia & Rampa & Comprimento (m) & Declividade média (\%) & Fator LS \\
\hline 1 & $1 \mathrm{a}$ & 67,7 & 15,0 & 2,53 \\
\hline 2 & $2 a$ & 131,0 & 10,4 & 2,67 \\
\hline 3 & $3 a$ & 128,9 & 14,6 & 6,02 \\
\hline 3 & $3 \mathrm{~b}$ & 69,1 & 23,0 & 4,75 \\
\hline 3 & $3 c$ & 110,3 & 15,5 & 4,49 \\
\hline 4 & $4 a$ & 24,8 & 11,0 & 2,47 \\
\hline 4 & $4 \mathrm{~b}$ & 72,8 & 10,3 & 2,33 \\
\hline
\end{tabular}


de contribuição específica, que resulta em valores mais baixos de fluxo acumulado de células de montante.

\section{AGRADECIMENTOS}

Ao Conselho Nacional de Desenvolvimento Científico e Tecnológico (CNPq) e ao SindiTabaco, pelo apoio financeiro.

\section{LITERATURA CITADA}

ARNOLD, J.G.; WILLIAMS, J.R.; NICKS, A.D. \& SAMMONS, N.B. SWRRB - A basin-scale simulation model for soil and water resources management. College Station, Texas A\&M Press, 1990. 195p.

DAVIS, J. Statistics and data analysis in geology. Toronto, John Wiley \& Sons, 1986. 646p.

DESMET, P.J. \& GOVERS, G. A GIS procedure for automatically calculating the USLE LS factor on topographically complex landscape units. J. Soil Water Conserv., 51:427-433, 1996.

FOSTER, G.R. \& WISCHMEIER, W.H. Evaluating irregular slopes for soil loss prediction. Trans. Am. Soc. Agric. Eng., 17:305-309, 1974.

FOSTER, G.R.; MEYER, L.D. \& ONSTAD, C.A. A runoff erosivity factor and variable slope length exponents for soil loss estimates. Trans. Am. Soc. Agric. Eng., 20:683687, 1977.

HADLEY, R.F.; LAL, R.; ONSTAND, C.A.; WALLING, D.E. \& YAIR, A. Erosion and sediment yield studies. Paris, UNESCO, 1985. 127p.

KINNELL, P. \& RISSE, L. USLE-M: Empirical modeling rainfall erosion throught runoff and sediment concentration. Soil Sci. Soc. Am. J., 62:1667-1672, 1998.

McCOOL, D.K.; BROWN, L.C.; FOSTER, G.R.; MUTCHLER, C.K. \& MEYER, L.D. Revised slope length factor for Universal Soil Loss Equation. Trans. Am. Soc. Agric. Eng., 32:1571-1576, 1989.

McCOOL, D.K.; FOSTER, G.R. \& WEESIES, G.A. Slope length and steepness factor. In: Predicting soil erosion by water - A guide to conservation planning with Revised Universal Soil Loss Equation (RUSLE). Washington, US Gov. Print Officie, 1997. (Agric. Handbook, 703)

MERRITT, W.S.; LETCHER, R.A. \& JAKEMAN, A.J. A review of erosion and sediment transport models. Environ. Mod. Soft., 18:761-799, 2003.

MINELLA, J.P.G.; MERTEN, G.H.; REICHERT, J.M. \& SANTOS, D.R. Identificação e implicações para a conservação do solo das fontes de sedimentos em bacias hidrográficas. R. Bras. Ci. Solo, 31:1637-1646, 2007.
MOORE, I.D. \& BURCH, G.J. Modeling erosion and deposition: Topographic effects. Trans. Am. Soc. Agric. Eng., 29:16241640, 1986a.

MOORE, I.D. \& BURCH, G.J. Sediment transport capacity of sheet and rill flow: Application of unit stream power theory. Water Res. Res., 22:1350-1360, 1986b.

MOORE, I.D. \& BURCH, G.J. Physical basis of the lengthslope factor in the universal soil loss equation. Soil Sci. Soc. Am. J., 50:1294-1298, 1986c.

MOORE, I. \& GALLANT, J. Overview of hydrologic and water quality modeling. In: MOORE, I., ed. Modelling the fate of chemicals in the environment. Camberra, Centre for Resource and Environmental Studies, Australian National University, 1991. p.1-8.

MOORE, I.D.; GRAYSON, R.B. \& LADSON, A.R. Digital terrain modelling: A review of hydrological, geomorfological and biological applications. Hydrol. Proc., 5:3-30, 1991.

ONSTAD, C.A. \& BRAKENSIEK, D.L. Watershed simulation by the stream path analogy. Water Res. Res., 4:965-971, 1968.

QUINN, P.F. The prediction of hillslope flow patchs for distributed hydrological modelling using digital terrain models. J. Hydrol. Proc., 5:59-79, 1991.

RENARD, K.G.; FOSTER, G.R.; WEESIES, G.A.; McCOOL, D.K. \& YODER, D.C. Predicting soil erosion by water - A guide to conservation planning with Revised Universal Soil Loss Equation (RUSLE). Washington, US Gov. Print Officie, 1997. (Agricultural Handbook, 703)

WALLING, D.E. The sediment delivery problem. J. Hydrol., 65:209-237, 1983.

WILLIAMS, J.R. \& BERNDT, H.D. Determining the universal soil loss equation's length-slope factor for waterscheds Soil erosion: Prediction and control. Soil Conserv. Am., 21:217-225, 1977. (Special Publication)

WILLIANS, J.R. Sediment routing for agricultural watersheds. Water Res. Bull., 11:965-974, 1975.

WISCHMEIER, W.H. \& SMITH, D.D. Predicting rainfall erosion losses: A guide to conservation planning. Washington, USDA, 1978. (Agricultural Handbook)

YANG, C.T. Unit stream power and sediment transport. J. Hydr. Div., Proc. Am. Soc. Civil Eng. 98:1805-1826, 1972.

YANG, C.T. Incipient motion and sediment transport J. Hydr. Div., Proc. Am. Soc. Civil Eng., 99:1679-1826, 1973.

YANG, C.T. Unit stream power equation for gravel J. Hydr. Div., Proc. Am. Soc. Civil Eng., 110:1783-1797, 1984.

YOUNG, R.A.; ONSTAD, C.A.; BOSCH, D.D. \& ANDERSON, W.P. AGNPS: A nonpoint-source pollution model for evaluating agricultural watersheds. J. Soil Water Conserv., 44:168-173,1989. 